\section{Inzidentelle Melanome meist in frühem Stadium}

\author{
Werden Patienten mit einer suspekten Hautläsion zu Dermatologen \\ überwiesen und entdecken die Hautspezialisten an anderer Stelle ein \\ Melanom, ist dieses meist kleiner und dünner als jene Melanome, \\ derentwegen die Konsultation erfolgt war.
}

$\mathrm{D}$ ermatologen aus den USA haben sich mit der Frage befasst, wie sich Melanome, die zufällig bei einer ausgedehnten Untersuchung anlässlich einer dermatologischen Konsultation gefunden werden, von jenen Fällen schwarzen Hautkrebses unterscheiden, die als verdächtige Läsionen die fachärztliche Diagnostik ursprünglich veranlasst hatten.

Dafür haben sie sich die Daten von mehr als 17.000 Überweisungen zu Dermatologen angesehen. 231 Melanome von 221 Patienten wurden dabei diagnostiziert. 144 davon hatten den Grund für die Überweisung geliefert, 87 - mehr als jedes dritte Melanom - wurden nebenbefundlich diagnostiziert. Die Wahrscheinlichkeit, dass ein Konsultationsmelanom invasiv wuchs, lag $51 \%$ höher als bei inzidentellen Melanomen. Die Melanome, derentwegen fachärztlicher Rat eingeholt wurde, hatten auch um $27 \%$ seltener eine Breslow-Dicke von weniger als $1,00 \mathrm{~mm}$. Insgesamt befanden sich $65,5 \%$ der inzidentellen, aber nur 38,9\% der Konsultationsmelanome in situ.

Generell waren inzidentelle Melanome kleiner als die Konsultationsläsionen, der mittlere Durchmesser betrug 0,98 $\mathrm{cm}$ gegenüber $1,3 \mathrm{~cm}$. Inzidentell entdeckte Malignome waren auch dünner, die Breslow-Dicke betrug im Mittel 0,64 $\mathrm{mm}$ im Vergleich zu 1,74 $\mathrm{mm}$ bei den Konsultationsmelanomen.

Melanom als Konsultationsdiagnose betraf am häufigsten den Kopf- und Hals- bereich (43,1\%), gefolgt vom Rumpfbereich $(31,9 \%)$ und den Extremitäten. Inzidentelle Melanome fanden sich hingegen besonders oft am Rumpf (44,8\%), wobei der Rücken die Hauptlokalisation war $(33,3 \%)$. 28,7 \% dieser Läsionen befanden sich in der Kopf-Hals-Region, 26,4\% an Schultern, Armen und Beinen.

Fazit: Melanome, die von Dermatologen diagnostiziert werden, haben ein früheres Stadium, so das Resümee der Autoren. Eine der Konsequenzen aus diesem Ergebnis sehen sie darin, Nichtdermatologen - Ärzte wie Patienten - besser im Aufspüren von Melanomen in früheren Stadien zu schulen. Doch auch Dermatologen sollten sich die Resultate zu Herzen nehmen: Diese unterstrichen, wie wichtig es sei, Patienten vollständig zu untersuchen und sich nicht nur die Konsulationsläsion anzusehen.

Dr. Robert Bublak

Hanson J L et al. Incidental melanomas detected in veterans referred to dermatology. J Am Acad Dermatol 2016; 74: 462-9

\title{
Nutzen der Immuntherapie beim Melanom unterschätzt?
}

\section{Möglicherweise wird bei Patienten mit einem Melanom, die mit Pembrolizumab behandelt werden, das Ansprechen unter Verwendung der traditionellen RECIST-Kriterien unterschätzt. Die Verwendung immunreaktionsspezifischer Kriterien könnte das vielleicht verhindern.}

In früheren Studien zur Immuntherapie beim Melanom gab es Hinweise, dass bei der Therapie mit Ipilimumab atypische Ansprechmuster auftreten. Selbst wenn es unter der Therapie zunächst zu einem Anstieg der Tumorlast oder zur Entstehung neuer Läsionen kommt, kann noch ein objektives Ansprechen möglich sein. US-Onkologen stellten deshalb die Hypothese auf, dass das auch bei Pembrolizumab der Fall ist und die Anwendung der RECIST-Kriterien („response evaluation criteria in solid tumors") nicht das ganze Bild des Ansprechens liefern.

Auf der Basis von Daten der Studie KEYNOTE-001mit 655 Melanompatienten prüften sie den Zusammenhang zwischen dem Gesamtüberleben und dem Ansprechen, beurteilt anhand der
RECIST-Kriterien sowie der immunassoziierten Responsekriterien (irRC). Die Muster unterscheiden sich in mehreren Punkten. Während bei RECIST als partielles Ansprechen gewertet wird, dass die Tumorlast um mindestens $30 \%$ im Vergleich zur Ausgangssituation abgenommen hat, wird nach den irRC-Kriterien eine Abnahme von mindestens $50 \%$ als solches gewertet. Unter RECIST werden nur maximal fünf Target-Läsionen gefordert, unter den irRC-Kriterien sind es maximal 15 Läsionen. Nicht zuletzt wird der Tumor unter den traditionellen Kriterien nur längs vermessen, unter irRC-Kriterien dagegen auch anhand der kurzen Tumormessachse. Beim Parameter komplettes Ansprechen sind sich beide gleich.
84 Patienten hatten eine Krankheitsprogression, wenn nach RECIST-Kriterien beurteilt wurde, nach irRC-Kriterien dagegen lag noch kein Fortschreiten vor. Bei ihnen betrug die Gesamtüberlebensrate nach zwei Jahren 37,5\% und war damit höher als bei den 177 Patienten, bei denen eine Krankheitsprogression anhand beider Kriterien gleichermaßen erkannt wurde $(17,3 \%)$.

Fazit: Ihren Berechnungen zufolge gehen die Autoren davon aus, dass in der Studie durch Verwendung der RECISTKriterien der Nutzen von Pembrolizumab um ungefähr $15 \%$ unterschätzt wurde. Es sei zu vermuten, dass die Patienten von der Immuntherapie auch dann noch profitierten, wenn bereits erste Zeichen einer radiografisch nachweisbaren Progression erkennbar seien. Die irRC-Kriterien könnten Therapeuten helfen, die Behandlung nicht vorzeitig abzubrechen.

Peter Leiner

Hodi FS et al. Evaluation of Immune-Related Response Criteria and RECIST v1.1 in Patients With Advanced Melanoma Treated With Pembrolizumab. J Clin Oncol 2016; 34: 1510-7 